\title{
Photoelectric Observations of AD Leo: 1989-1994
}

\author{
R. Konstantinova-Antova, A.P. Antov
}

Institute of Astronomy, Bulgarian Academy of Sciences, 72 Tsarigradsko shose, BG-1784 Sofia, Bulgaria

\section{Observations}

An investigation of the activity of the flare star AD Leo was made using $U$-band patrol observations. $60 \mathrm{~cm}$ computer-controlled telescopes with identical single channel photon-counting photometers at the National Astronomical Observatory at Rozhen and at the Belogradchik Observatory were used. The integration time was $1 \mathrm{sec}$. Differential photometry was carried out, the AD Leo measurements were made relative to $\mathrm{BD}+20^{\circ} 2475$. The data was reduced with the program package APR (Kirov et al. 1991).

Two simultaneous observing runs were made. The results of the first have already been published (Antov et al. 1991). The other was part of the observational campaign in May, 1991 with the ROSAT satellite. In Bulgaria, the observations were carried out at Rozhen in the $U$-band and at Belogradchik in the $B$-band. Four flares were detected by both.

\section{Results}

37 flares with amplitudes more than $4 \sigma$ were detected during $71^{\mathrm{h}} 05^{\mathrm{m}} 17^{\mathrm{s}}$ total monitoring time. Approximately $16 \%$ are so called fast flares, with a duration $<1 \mathrm{~min}$. If flares with amplitude $<4 \sigma$ are added, the fast flares are $\sim 23 \%$. Usually the fast flares had an amplitude $\leq 0.5$ (except one flare, detected on 29.3.1993 with an amplitude 0.72 ).

The reality of so-called single spikes on $A D$ Leo, with a duration 1-2 sec was not confirmed during the period of the observations. Several such events were detected at Rozhen (altitude $1750 \mathrm{~m}$ ) but none were detected at Belogradchik (altitude $630 \mathrm{~m}$ ). One possible explanation is that the spikes were due to cosmic rays or artificial sources.

A rough investigation of the flare activity of AD Leo was carried out. The rate of the flare activity (the number of flares $\mathrm{hr}^{-1}$ ) was calculated for each year of the period (Table 1) and the result is shown in Fig. 1. 
Table 1. Rate of the flare activity of AD Leo

\begin{tabular}{lcccc}
\hline Year & $\begin{array}{c}\text { No. of } \\
\text { nights }\end{array}$ & $\begin{array}{c}\text { Effective } \\
\text { monitoring time }\end{array}$ & $\begin{array}{c}\text { No. of } \\
\text { flares }\end{array}$ & Flares hr \\
\hline 1989 & 2 & $2^{\mathrm{h}} 51^{\mathrm{m}} 45^{\mathrm{s}}$ & 2 & $0.95 \pm 0.47$ \\
1990 & 15 & $25^{\mathrm{h}} 47^{\mathrm{m}} 00^{\mathrm{s}}$ & 18 & $0.70 \pm 0.16$ \\
1991 & 10 & $12^{\mathrm{h}} 53^{\mathrm{m}} 12^{\mathrm{s}}$ & 7 & $0.58 \pm 0.20$ \\
1992 & 10 & $14^{\mathrm{h}} 59^{\mathrm{m}} 30^{\mathrm{s}}$ & 5 & $0.33 \pm 0.15$ \\
1993 & 4 & $3^{\mathrm{h}} 25^{\mathrm{m}} 08^{\mathrm{s}}$ & 1 & $0.29 \pm 0.28$ \\
1994 & 7 & $11^{\mathrm{h}} 08^{\mathrm{m}} 42^{\mathrm{s}}$ & 4 & $0.36 \pm 0.18$ \\
\hline
\end{tabular}

It appears that the rate of flare activity changes from year to year in a systematic way. This fact could be explained by the existence of a change in the chromospheric activity on a long time scale.

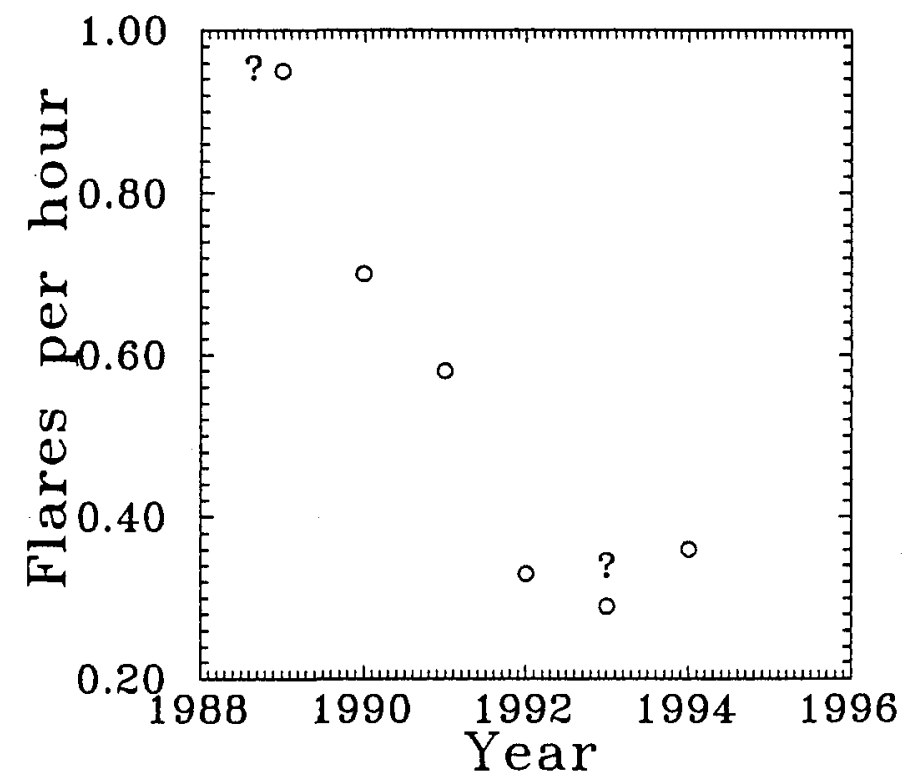

Fig. 1. Flare activity rate of AD Leo.

Acknowledgements: R.K.-A. is thankful to the German Science Foundation for the financial support to attend the Colloquium.

\section{References}

Antov A., Genkov V., Konstantinova-Antova R., Kirov N., 1991, IBVS 3577

Kirov N.K., Antov A.P., Genkov V.V., 1991, Compt. Rend. Bulg. Acad. Sci. 44, 5 